\title{
The COVID-19 Host Genetics Initiative, a global initiative to elucidate the role of host genetic factors in susceptibility and severity of the SARS-CoV-2 virus pandemic
}

\author{
The COVID-19 Host Genetics Initiative ${ }^{1,2}$
}

Received: 14 April 2020 / Accepted: 17 April 2020 / Published online: 13 May 2020

(c) The Author(s), under exclusive licence to European Society of Human Genetics 2020. This article is published with open access

\section{Introduction}

The COVID-19 pandemic is a global crisis creating severe disruptions across the economy and health system. Insights into how to better understand and treat COVID-19 are desperately needed.

Early studies have focused on the clinical characteristics [1-3], epidemiology $[1,4,5]$, and genomic characterization [6-8] of SARS-CoV-2 infection. These studies have also highlighted the value and importance of transparent data sharing across countries, which have enabled the live tracking of the disease widespread worldwide $[9,10]$. The role of host genetics in impacting susceptibility and severity of COVID-19 has been less studied. Previous work has supported the role of human leukocyte antigen (HLA) in susceptibility [11] and severity [12] for several viral infections. Moreover, a synonymous variant in the IFN-induced transmembrane protein-3 gene has been reported to cause severe clinical outcomes in patients infected with H7N9 and H1N1 influenza viruses [13, 14], although results did not reach established $P$ value thresholds $\left(P<5 \times 10^{-8}\right)$. In addition, candidate variant studies have suggested host factors that are critical for severe disease in other coronavirus infections, such as infections due to the related SARS-CoV [15].

Given the importance and urgency of exploring the role of the host genome in conjunction with COVID-19 clinical and genomic variability, and the recognition that this can only be achieved with the combined effort of the scientific community, we launched the 'COVID-19 Host Genetics Initiative'. This initiative brings together the human

Andrea Ganna

aganna@broadinstitute.org

1 Institute for Molecular Medicine Finland, University of Helsinki, Helsinki, Finland

2 Analytical and Translational Genetic Unit, Massachusetts General Hospital, Harvard Medical School, Boston, MA, USA genetics community to generate, share, and analyze data to learn the genetic determinants of COVID-19 susceptibility, severity, and outcomes. Such discoveries could help to identify individuals at unusually high or low risk, generate hypotheses for drug repurposing, and contribute to global knowledge of the biology of SARS-CoV-2 infection and disease. The initiative has three main goals:

1. Provide an environment to foster the sharing of resources to facilitate COVID-19 host genetics research (e.g., protocols, questionnaires).

2. Organize analytical activities across studies to identify genetic determinants of COVID-19 susceptibility and severity.

3. Provide a platform to share the results from such activities, as well as the individual-level data where possible, to benefit the broader scientific community.

\section{Approach}

The COVID-19 host genetics initiative is a bottom-up initiative with a flexible, decentralized structure that is based on the following collaborative principles:

1. Collaborate in an environment of honesty, fairness, and trust

2. Promote early-career researchers

3. Respect other groups' data

4. Operate transparently with a goal of no surprises

5. Seek permission from each group to use results prior to public release

6. Do not share another group's results with other parties without permission

7. The initiative should not inhibit any work being done within any individual studies (or between pairs of studies). 
Studies that are interested in joining the initiative can register via the website ${ }^{1}$. We can categorize the participating studies in two main groups. Retrospective collections are typically biobanks with existing significant genetic data and active connections to health systems. In these studies, there is the opportunity to opportunistically and rapidly develop a genetic study on susceptibility and severity. For example, in Finland with the national network of biobanks covering each hospital district, it is possible to acquire almost 'real-time' updates on COVID-19 status of individuals that are already part of the FinnGen study ${ }^{2}$. This group of studies is already connected and loosely structured via other initiatives such as the Global Biobank Metaanalysis Initiative ${ }^{3}$.

The second group of studies includes prospective collection that have recently started to directly consent incoming COVID-19 patients. More than just the critical jump in scale for studying progression, severity, and outcomes, these studies bring important additional opportunities not only for deeper DNA studies, but potentially informative viral and antibody profiling and epitope mapping experiments which can be implemented in many sites with relatively small blood/plasma requirements.

\section{Data sharing}

We expect that a sizable fraction of the studies will be able to share individual-level data. Genetics and clinical data are submitted to the European Genome-phenome Archive (EGA) under controlled access, and this is coordinated with viral sequence deposition efforts and coordination of other biomolecular data with EU, EOSC, ELIXIR, and other institutions across the globe. Alternatively, studies are able to share summary statistics, which will be directly made available on the website and via the GWAS catalog [16].

The majority of the planning, discussion, and exchange of information between the participants study, analysts, and clinicians is done on a dedicated Slack workspace with the support of the International Common Disease Alliance $(\mathrm{ICDA})^{4}$.

\section{Phenotype and analysis}

The initiative aims to support widespread sharing of data and knowledge across participants groups. Groups can connect and initiate collaborations focused on specific phenotypes. Few analyses that can benefit from maximal sample size are centralized. The primary analysis focuses on

\footnotetext{
${ }^{1}$ https://www.covid19hg.org/register/

${ }^{2}$ https://www.finngen.fi/en

3 https://www.globalbiobankmeta.org/

4 https://www.icda.bio/
}

COVID-19 disease severity. There are challenges in defining COVID-19 severity across multiple studies and healthcare systems. We used a pragmatic approach which considers the use of invasive and noninvasive ventilation as an index of severity. The advantages of this approach is the possibility to easily retrieve this information from electronic health records and the widespread use of these procedures across healthcares. Studies that have collected detailed clinical information can perform secondary analyses using continuous markers of disease severity such as maximum respiratory rate during hospitalization or prior to invasive respiratory support.

Bioinformatic and statistical analysis will consider data generated from GWAS array, exome and genome sequencing, leveraging the impact of both common and rare variants. Key analysis will take into account differences between sexes, ancestries, and date of sample collection. The latter aspect is important to consider given the rapid changes in population screening procedures and hospital capacity with consequent impact on the severity of patients included in different studies.

Given the importance of the HLA genes system for the etiology of infectious diseases and autoimmune disorders, we will impute classical HLA alleles and the corresponding amino acid sequences. COVID indiscriminately affects populations from all around the world, and HLA variation is specific to different populations. Hence, we propose using a multiethnic HLA reference panel constructed using deepcoverage whole-genome sequencing data from 21,546 individuals of five different populations: European, African, Latino, Asian, and South Asian. This reference panel will capture much of the HLA variation around the world. This will allow to test each HLA allele and also each of the amino acid site position within HLA genes to assess if they explain COVID risk.

\section{Participant studies}

At time of writing 105 studies have joined the initiative, and participation is still expanding. The majority of studies are conducted in Europe (55\%) and the US (28\%), amongst which the United Kingdom (10\%) and Italy (9\%) are the largest. However there are also participants from Asia (Republic of Korea and Malaysia), Australia, the Middle-East (Kuwait, Pakistan, and Qatar), and Africa (Nigeria); Fig. 1, an updated list is available on the website ${ }^{5}$. Most studies (71\%) have initiated a new prospective collection, $27 \%$ have done that on top of existing retrospective collections. Array-based genotyping is the most common approach, considered by $69 \%$ of the participant studies, while exome and genome sequencing are less common, (29\%). Antibody and immune

\footnotetext{
${ }^{5}$ https://www.covid19hg.org/partners/
} 


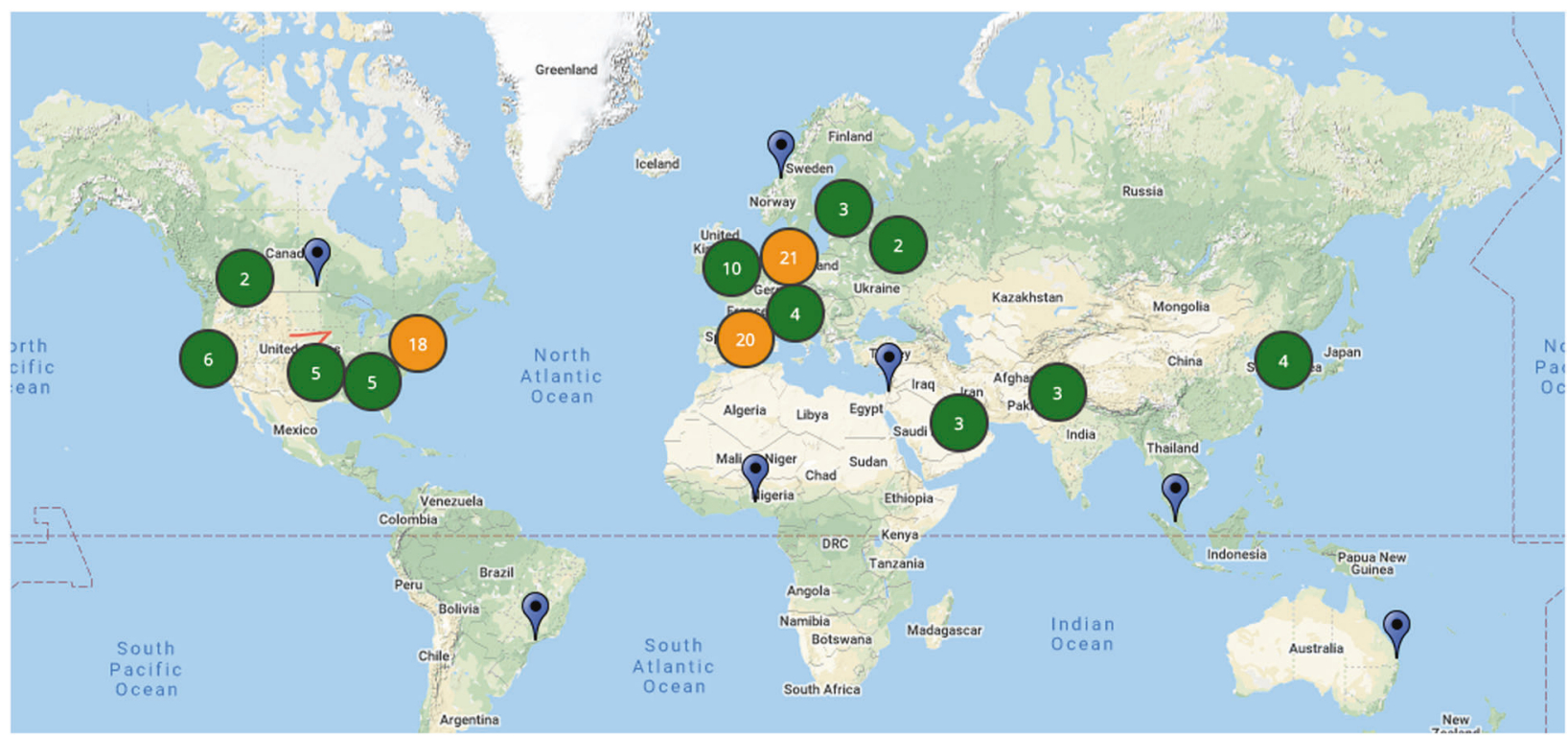

Fig. 1 Map of the studies registered to the initiative by 13th of April 2020. The map report aggregate counts of studies registered to the COVID-19 Host Genetics Initiative.

profiling are the two most common additional assays that are reported by the contributing studies.

\section{Conclusion}

We initiated a global effort to study the relationship between host genome and SARS-CoV-2 infection. Our approach is inclusive, decentralized, and transparent. While providing novel scientific insights remains a priority of the initiative, we equally value the creation of an infrastructure that facilitates communication between studies with similar scientific goals. We expect the COVID-19 host genetics initiative to substantially contribute to the understanding of the variability of COVID-19 susceptibility, severity, and outcomes in the population within the next few months.

Acknowledgements We want to thank all the study participants that have donated-and still are donating-samples to help research on COVID-19. The COVID-19 host genetics initiative was originally initiated by AG and Mark Daly, but it belongs to all the participant studies. Because a definite list of studies and contributing scientists is not yet available, we decided to not include any one specific author in this article. We want to thank Yang Luo for contributing with the HLA imputation panel and Ewan Birney, Thomas Keane for their guidance on data sharing.

\section{Compliance with ethical standards}

Conflict of interest The authors declare that they have no conflict of interest.

Publisher's note Springer Nature remains neutral with regard to jurisdictional claims in published maps and institutional affiliations.
Open Access This article is licensed under a Creative Commons Attribution 4.0 International License, which permits use, sharing, adaptation, distribution and reproduction in any medium or format, as long as you give appropriate credit to the original author(s) and the source, provide a link to the Creative Commons license, and indicate if changes were made. The images or other third party material in this article are included in the article's Creative Commons license, unless indicated otherwise in a credit line to the material. If material is not included in the article's Creative Commons license and your intended use is not permitted by statutory regulation or exceeds the permitted use, you will need to obtain permission directly from the copyright holder. To view a copy of this license, visit http://creativecommons. org/licenses/by/4.0/.

\section{References}

1. Huang C, Wang Y, Li X, Ren L, Zhao J, Hu Y, et al. Clinical features of patients infected with 2019 novel coronavirus in Wuhan, China. Lancet. 2020;395:497-506.

2. Deng Y, Liu W, Liu K, Fang Y-Y, Shang J, Zhou L, et al. Clinical characteristics of fatal and recovered cases of coronavirus disease 2019 (COVID-19) in Wuhan, China: a retrospective study. Chin Med J. 2020. https://doi.org/10.1097/CM9.0000000000000824.

3. Zhou F, Yu T, Du R, Fan G, Liu Y, Liu Z, et al. Clinical course and risk factors for mortality of adult inpatients with COVID-19 in Wuhan, China: a retrospective cohort study. Lancet. 2020;395:1054-62.

4. Onder G, Rezza G, Brusaferro S. Case-fatality rate and characteristics of patients dying in relation to COVID-19 in Italy. JAMA. 2020. https://doi.org/10.1001/jama.2020.4683

5. Chan JF-W, Yuan S, Kok K-H, To KK-W, Chu H, Yang J, et al. A familial cluster of pneumonia associated with the 2019 novel coronavirus indicating person-to-person transmission: a study of a family cluster. Lancet. 2020;395:514-23.

6. Lu R, Zhao X, Li J, Niu P, Yang B, Wu H, et al. Genomic characterisation and epidemiology of 2019 novel coronavirus: implications for virus origins and receptor binding. Lancet. 2020;395:565-74. 
7. Zhou P, Yang X-L, Wang X-G, Hu B, Zhang L, Zhang W, et al. A pneumonia outbreak associated with a new coronavirus of probable bat origin. Nature. 2020;579:270-3.

8. Gudbjartsson DF, Helgason A, Jonsson H, Magnusson OT, Melsted P, Norddahl GL, et al. Early spread of SARS-Cov-2 in the Icelandic Population. Epidemiology. 2020. https://doi.org/10. 1101/2020.03.26.20044446.

9. WHO. Novel Coronavirus (2019-nCoV) situation reports. 2020. https://www.who.int/emergencies/diseases/novel-coronavirus2019/situation-reports/.

10. Dong E, Du H, Gardner L. An interactive web-based dashboard to track COVID-19 in real time. Lancet Infect Dis. 2020. https://doi. org/10.1016/S1473-3099(20)30120-1.

11. Tian C, Hromatka BS, Kiefer AK, Eriksson N, Noble SM, Tung JY, et al. Genome-wide association and HLA region fine-mapping studies identify susceptibility loci for multiple common infections. Nat Commun. 2017;8:599.

12. International HIV Controllers Study, Pereyra F, Jia X, McLaren PJ, Telenti A, de Bakker PIW, et al. The major genetic determinants of HIV-1 control affect HLA class I peptide presentation. Science. 2010;330:1551-7.

13. Wang Z, Zhang A, Wan Y, Liu X, Qiu C, Xi X, et al. Early hypercytokinemia is associated with interferon-induced transmembrane protein-3 dysfunction and predictive of fatal H7N9 infection. Proc Natl Acad Sci USA. 2014;111:769-74.

14. Everitt AR, Clare S, Pertel T, John SP, Wash RS, Smith SE, et al. IFITM3 restricts the morbidity and mortality associated with influenza. Nature. 2012;484:519-23.

15. Ching JC-Y, Chan KYK, Lee EHL, Xu M-S, Ting CKP, So TMK, et al. Significance of the myxovirus resistance A $(\mathrm{MxA})$ gene $-123 \mathrm{C}>\mathrm{a}$ single-nucleotide polymorphism in suppressed interferon beta induction of severe acute respiratory syndrome coronavirus infection. J Infect Dis. 2010;201: 1899-908.

16. MacArthur J, Bowler E, Cerezo M, Gil L, Hall P, Hastings E, et al. The new NHGRI-EBI Catalog of published genome-wide association studies (GWAS Catalog). Nucleic Acids Res. 2017;45: D896-901. 